\title{
Association of diabetes with severity and mortality in hospitalized patients with COVID-19 in Wuhan, China: a single-centered, retrospective study
}

\begin{abstract}
'Department of Pediatrics, Zhongnan Hospital of Wuhan University, Wuhan, P.R. China

2 Department of Clinical Laboratory, Zhongnan Hospital of Wuhan University, Wuhan, P.R. China ${ }^{3}$ Department of Urology,

Zhongnan Hospital of Wuhan University, Wuhan, P.R. China ${ }^{4}$ State Key Laboratory of Virology/ Institute of Medical Virology, School of Basic Medical Sciences, Wuhan University, Wuhan, P.R. China
\end{abstract}

${ }^{\S}$ These authors contributed equally to this work.

\author{
Correspondence to: \\ Xue-dong $\mathrm{Fu}$ \\ Department of Pediatrics, Zhongnan \\ Hospital of Wuhan University \\ 169 Donghu Road, 430071, Wuhan, \\ China \\ wb001865@whu.edu.cn \\ Hai-rong Xiong \\ Institute of Medical Virology, Schoo \\ of Basic Medical Sciences, Wuhan \\ University \\ 185 Donghu Road, 430071, Wuhan, \\ China \\ hrxiong@whu.edu.cn \\ Received on Sept/20/2020 \\ Accepted on Apr/22/2021 \\ DOI: 10.20945/2359-3997000000384
}

\author{
You-ping Deng ${ }^{1 \S}$ \\ https://orcid. org/0000-0003-2002-684 \\ Wen $\mathrm{Xie}^{2 \S}$ \\ https://orcid.org/0000-0002-4673-9711 \\ Tao Liu $^{3}$ \\ https://orcid. org/0000-0002-4202-8218 \\ Shou-yi Wang' \\ https://orcid.org/0000-0002-6139-3608 \\ Yu-xing Zan ${ }^{4}$ \\ https://orcid.org/0000-0001-9551-2137 \\ Mei-rong Wang ${ }^{4}$ \\ https://orcid.org/0000-0003-1099-1891 \\ Xiao-bo Meng ${ }^{1}$ \\ https://orcid.org/0000-0001-5056-2253 \\ Jie Zheng \\ https://orcid.org/0000-0003-4105-9343 \\ Hai-rong Xiong ${ }^{4}$ \\ https://orcid.org/0000-0002-4028-0639 \\ Xue-dong $\mathrm{Fu}^{1}$ \\ https://orcid.org/0000-0003-4335-8291
}

\begin{abstract}
Objective: Coronavirus disease 2019 (COVID-19), caused by severe acute respiratory syndrome coronavirus 2 (SARS-CoV-2), has spread worldwide. The aim this study was to investigate the association of diabetes with severity and mortality among hospitalized patients with COVID-19 in Wuhan, China. Subjects and methods: This retrospective, single-center case study enrolled a total of 564 patients diagnosed with COVID-19 at the Seventh Hospital of Wuhan City, between January 20 and March 15, 2020. Results: Among the 564 patients with confirmed COVID-19, 509 (85.1\%) were discharged and 55 (9.8\%) died. The median age was 59 years (range, $10-93$ years). A total of 85 (15.1\%) patients were diagnosed with diabetes on admission (median age, 65.0 [range, 34-91] years). Patients with diabetes had significantly higher proportions of critical cases (24 [28.2\%] vs. 66 [13.8\%]) and in-hospital mortality (17 [20\%] vs. 38 [7.9\%]). Moreover, patients with diabetes presented abnormal levels of multiple indicators concerning lymphopenia, inflammation, heart, liver, kidney, and lung function on admission, while diabetic patient group still display higher troponin T (TnT) levels when approaching discharge. The Kaplan-Meier survival curve indicated a trend toward poorer survival in diabetic patients compared to non-diabetic patients, also evidenced by abnormal laboratory biomarker changes regarding multiple system impairments among COVID-19 patients with diabetes with in-hospital death. Conclusion: The detailed clinical investigation of 564 hospitalized patients with COVID-19 indicated a considerable association between diabetes and COVID-19 severity or mortality. Thus, more intensive treatment may be considered for COVID-19 patients with diabetes, especially regarding to cardiac injury. Arch Endocrinol Metab. 2021;65(5):596-608
\end{abstract}

\section{Keywords}

COVID-19; diabetes, mortality; clinical characteristics

\section{INTRODUCTION}

Tovel coronavirus disease 2019 (COVID-19) is 1 caused by the severe acute respiratory syndrome coronavirus 2 (SARS-CoV2), which is a newly emerged envelope RNA $\beta$-coronavirus with a round/oval shape and a diameter of 60-140 nm $(1,2)$. The COVID-19 outbreak has spread worldwide, endangering global public health $(3,4)$. SARS-CoV2 infection can induce 
clinical symptoms including fever, dry cough, dyspnea, and fatigue, and ultimately result in acute respiratory distress syndrome (ARDS), septic shock, and multiple organ dysfunction syndrome with high morbidity and mortality (1). Early reports suggested that patients with certain comorbid conditions, such as hypertension, cardiovascular disease, and diabetes, may face higher risks and are more likely to develop severe COVID-19 $(3,5)$.

Diabetes mellitus is a complex chronic illness that is associated with considerable morbidity and mortality worldwide (6). Patients with diabetes are more sensitive to infections and may have a poor prognosis compared to that in patients without diabetes, which may be due to the impairment of their immune status (7). Diabetes has been reported to be one of the most frequent comorbidities among patients with COVID-19 $(8,9)$. Therefore, we retrospectively reviewed the clinical data from a single center in Wuhan, China, and assessed the association between diabetes and COVID-19. We compared the differences in clinical characteristics, laboratory findings, treatment, and outcomes between diabetic patients and non-diabetic patients, as well as those indicators among the survivors and non-survivors in patients with diabetes, which may provide a hint for the clinical management of diabetic patients with COVID-19.

\section{SUBJECTS AND METHODS}

\section{Study design}

This study was conducted at the No.7 Hospital of Wuhan, a government-designated that was consigned to Zhongnan Hospital of Wuhan University during the COVID-19 pandemic. A total of 564 patients with confirmed COVID-19 hospitalized at the No.7 Hospital of Wuhan were enrolled in this study, which was conducted from January 20 to March 15, 2020. All patients were diagnosed with COVID-19 and classified into distinct clinical types according to the diagnostic and treatment guidelines of COVID-19 from the Chinese National Health Commission (version 3-7) (10). According to the National Public Health Emergency Management System, patients with mild-type COVID-19 were treated at Fangcang shelter hospitals $(11,12)$. Thus, all patients involved in this study were in moderate, severe, or critical condition. Patients with diabetes were verified through medical records or self-reported diagnoses reviewed by their physicians. This study was approved by the institutional ethics board of Zhongnan Hospital, Wuhan University (No.2020056K), which waived the requirement for written informed consent for the emerging infectious disease.

\section{Data collection}

The medical records, including basic information (age, sex, comorbidities, etc.), clinical characteristics, laboratory findings, radiological examinations, treatment, and outcomes of each patient were obtained from their medical records. The date of disease onset was defined as the day on which symptoms were noticed. Pharyngeal swab specimens were collected for laboratory viral nucleic acid detection of SARS-CoV-2 using quantitative reverse-transcription-polymerase chain reaction (qRT-PCR) (13). The patient samples were also tested for other viral pathogens, including influenza virus, parainfluenza, Coxsackie virus, adenovirus, echovirus, respiratory syncytial virus, and cytomegalovirus. All patients underwent chest computed tomography (CT) or X-ray radiography. Follow-up radiological examination and negative SARS-CoV-2 test results were considered reference indices for cure and hospital discharge.

Laboratory examinations conducted at admission and with disease progression included routine blood tests and assessments of blood biochemistry, blood gas level, blood electrolytes, coagulation function, procalcitonin (PCT), C-reactive protein (CRP), serum amyloid A (SAA), serum creatine kinase and myocardial enzyme spectrum. Medical treatments were recorded, including antiviral treatment, Chinese patent medicine, corticosteroids, gamma globulin, probiotics, etc. Treatment strategies, such as supplemental oxygen, noninvasive mechanical ventilation, or invasive mechanical ventilation, were also recorded.

\section{Statistical analysis}

Non-normally distributed continuous data were described using median and interquartile range (IQR) values, while categorical data were expressed as numbers/frequencies and percentages. Chi-square and Fisher's exact tests were used to compare the frequencies of the categorical variables. Continuous variables were tested for Gaussian distribution by D'Agostino-Pearson omnibus normality test and further analyzed using Mann-Whitney tests as appropriate. The association 
between diabetes and death was quantified by logic regression after adjusting for controlled confounders. The E-values were then calculated to assess the robustness of the associations to potential unmeasured confounders using "EValue" package in software $\mathrm{R}$ (14). Survival curves were generated using the KaplanMeier method, and comparisons between groups were performed using log-rank tests. Landmark analyses were performed using EmpowerStats (http://www. empowerstats.com) and the statistical package R. Other statistical analyses were performed using GraphPad Prism version 6.00 software (GraphPad Software Inc.). $P$ value less than .05 was considered statistically significant.

\section{RESULTS}

\section{Demographics and clinical characteristics}

The flowchart in Figure 1 shows the patient selection in this study. Briefly, a total 630 of patients in the medical record system were screened from January 20 to March 15,2020 , among which 40 patients were not confirmed and 26 patients without core medical information and duplicated records were excluded. Thus, the present study included a total of 564 patients hospitalized with confirmed COVID-19, including 85 (15.1\%) patients diagnosed with diabetes on admission. The median age of all patients was 59 years (range, $10-93$ years), and $275(50.7 \%)$ patients were male. The most common

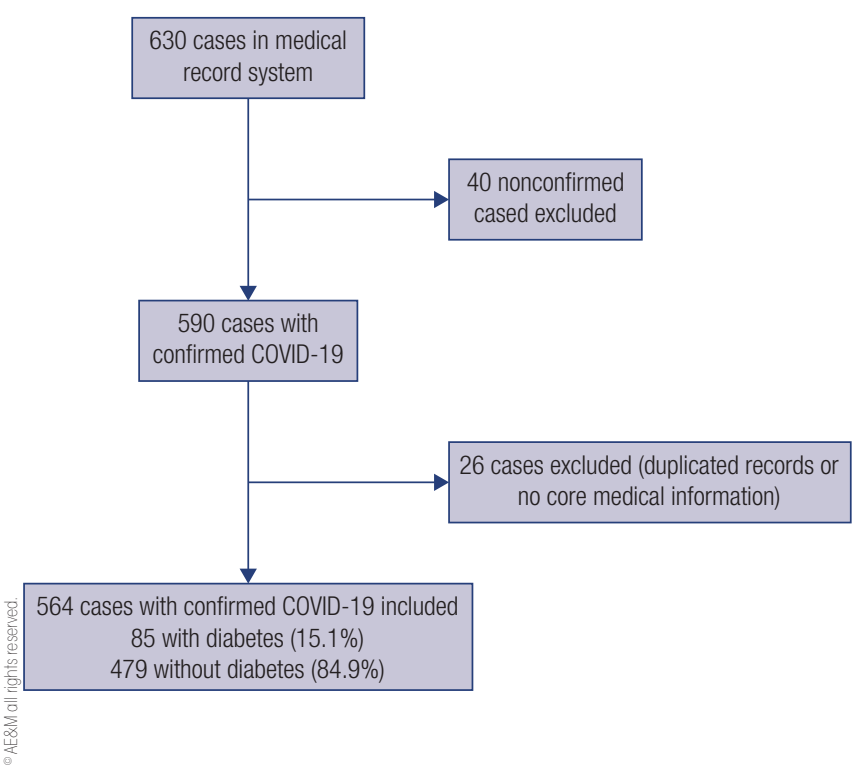

Figure 1. Flowchart of patient recruitment. underlying comorbidities were hypertension $(\mathrm{n}=190$, $33.7 \%)$, cardiovascular disease $(\mathrm{n}=70,12.4 \%)$, and liver disease $(\mathrm{n}=33,5.9 \%)$. Among the 564 patients, 284 $(50.4 \%), 190(33.7 \%)$, and $90(16.0 \%)$ were categorized as having moderate, severe, and critical statuses. Of these 564 patients, $509(90.2 \%)$ were discharged and $55(9.8 \%)$ died.

Compared to patients without diabetes, patients with diabetes were older (median [range] age, 65 [34$91]$ vs. 57 [10-93] years; $p<0.0001)$ and most were male $(49,57.6 \%)$. Moreover, patients with diabetes presented significantly higher rates of comorbidities, including hypertension (58 [68.2\%] vs. 132 [27.6\%], $p<0.0001)$ and cardiovascular disease (25 [29.4\%] vs. $45[9.4 \%], p<0.0001)$. Patients with diabetes had a significantly higher proportion of critical cases $(24$ [28.2\%] vs. $66[13.8 \%], p=0.0019)$. Mortality was also significantly higher in patients with diabetes (17 [20.0\%] vs. 38 [7.9\%]) (Table 1).

\section{Laboratory findings on admission}

As shown in Table 2, in the overall study population of 564 patients, the median levels of C-reactive protein (CRP) $(20.30$ [2.50-59.59] mg/L), and serum amyloid A $(74.9[12.0-173.1] \mathrm{mg} / \mathrm{L})$ were elevated, while those of lymphocyte count $\left(1.02[0.65-1.50] \times 10^{9} / \mathrm{L}\right)$, total protein $(64.1[60.3-67.8] \mathrm{g} / \mathrm{L})$, and albumin (37.4 [33.1-40.8] g/L) were decreased. However, the other laboratory indicators were within the normal ranges, including other blood cell counts, blood lipids and electrolytes, cardiac biomarkers, blood gas analysis, and other biomarkers of liver and renal function.

Compared to patients without diabetes, patients with diabetes presented with significantly higher neutrophil counts (median [IQR], 3.88 [3.00-6.01] vs. $\left.3.17[2.31-4.80] \times 10^{9} / \mathrm{L}, p=0.002\right)$, and lower lymphocyte counts (median [IQR], 0.82 [0.60-1.34] vs. $\left.1.05[0.67-1.53] \times 10^{9} / \mathrm{L} ; p=0.0153\right)$. The white blood cell, lymphocyte, and platelet counts of these two groups were similar.

Triglyceride and small dense low-density lipoprotein (sdLDL) levels did not differ between patients with and without diabetes, while patients with diabetes had lower levels of total cholesterol (median [IQR], 3.38 [2.87$4.35]$ vs. 3.89 [3.00-4.32] mmol/L; $p=0.0206$ ), highdensity lipoprotein (HDL) (median [IQR], 1.08 [0.84$1.24]$ vs. 1.14 [0.97-1.32] mmol $/ \mathrm{L} ; p=0.0055)$, and low-density lipoprotein (LDL) (median [IQR], 1.92 
Table 1. Demographics and clinical characteristics of patients with COVID-19

\begin{tabular}{|c|c|c|c|c|}
\hline \multirow[b]{2}{*}{ Characteristic } & \multicolumn{4}{|c|}{ No. (\%) } \\
\hline & $\begin{array}{c}\text { Total } \\
(n=564)\end{array}$ & $\begin{array}{l}\text { Non-diabetes } \\
(n=479)\end{array}$ & $\begin{array}{c}\text { Diabetes } \\
(n=85)\end{array}$ & $P$ value \\
\hline Age-median(range) & $59(10-93)$ & $57(10-93)$ & $65(34-91)$ & $<0.0001$ \\
\hline \multicolumn{5}{|l|}{ Sex } \\
\hline Female & $289(51.2)$ & $253(52.8)$ & $36(42.4)$ & 0.0786 \\
\hline Male & $275(48.8)$ & $226(47.2)$ & $49(57.6)$ & \\
\hline Smoking & $42(7.4)$ & 38 & 4 & 0.3745 \\
\hline Onset of symptom to hospital admission, median (IQR), d & $10(6-15)$ & $10(6-15)$ & $10(7-15)$ & 0.7271 \\
\hline Hospitalization, median (IQR), d & $15(9-24)$ & $15(9-23)$ & $17(10-25)$ & 0.3302 \\
\hline \multicolumn{5}{|l|}{ Comorbidity - No. (\%) } \\
\hline Cardiovascular disease & $70(12.4)$ & $45(9.4)$ & $25(29.4)$ & $<0.0001$ \\
\hline Cerebrovascular disease & $11(2.0)$ & $7(1.5)$ & $4(4.7)$ & 0.0685 \\
\hline Hypertension & $190(33.7)$ & $132(27.6)$ & 58 (68.2) & $<0.0001$ \\
\hline Chronic bronchitis & $12(2.1)$ & $11(2.3)$ & $1(1.2)$ & 1 \\
\hline Malignancy & $24(4.3)$ & $18(3.8)$ & $6(7.1)$ & 0.2364 \\
\hline Liver disease & $33(5.9)$ & $29(6.1)$ & $4(4.7)$ & 0.8038 \\
\hline Kidney disease & $25(4.4)$ & $19(4.0)$ & $6(7.1)$ & 0.2461 \\
\hline Allergic physique & $19(3.4)$ & $15(3.1)$ & $4(4.7)$ & 0.5092 \\
\hline \multicolumn{5}{|l|}{ Complication } \\
\hline Bacterial infection & $42(7.4)$ & $35(7.3)$ & $7(8.2)$ & 0.8220 \\
\hline Metabolic acidosis & $19(3.4)$ & $11(2.3)$ & $8(9.4)$ & 0.0036 \\
\hline Heart failure & $25(4.4)$ & $15(3.1)$ & $10(11.8)$ & 0.0017 \\
\hline ARDS & $61(10.8)$ & $41(8.6)$ & $20(23.5)$ & 0.0002 \\
\hline Acute liver injury & $24(4.3)$ & $18(3.8)$ & $6(7.1)$ & 0.2364 \\
\hline Acute kidney injury & $27(4.8)$ & $19(4.0)$ & $8(9.4)$ & 0.0477 \\
\hline DIC & $7(1.2)$ & $3(0.6)$ & $4(4.7)$ & 0.0118 \\
\hline \multicolumn{5}{|l|}{ Treatments } \\
\hline Antiviral treatment & 351 (62.2) & 299 (62.4) & 52 (61.2) & 0.9034 \\
\hline Antibiotics & $407(72.1)$ & 341 (71.1) & 66 (77.6) & 0.2397 \\
\hline Chinese Medicine & $343(60.8)$ & $289(60.3)$ & 54 (63.5) & 0.6305 \\
\hline Glucocorticoid & 193 (34.2) & 159 (33.2) & $34(40.0)$ & 0.2641 \\
\hline Immune globulin & $90(16.0)$ & 70 (14.6) & $20(23.5)$ & 0.0524 \\
\hline Respiratory support & & & & 0.0462 \\
\hline Nasal cannula & $305(54.1)$ & $257(53.7)$ & $48(56.5)$ & \\
\hline Non-invasive ventilation & $35(6.2)$ & $25(5.2)$ & $10(11.8)$ & \\
\hline Invasive ventilation & $15(2.7)$ & $10(2.1)$ & $5(5.9)$ & \\
\hline Diseases severity & & & & 0.0018 \\
\hline Moderate & $284(50.4)$ & $252(52.6)$ & $32(37.6)$ & \\
\hline Severe & $190(33.7)$ & $161(33.6)$ & $29(34.1)$ & \\
\hline Critical & $90(16.0)$ & 66 (13.8) & 24 (28.2) & \\
\hline Clinical outcomes & & & & 0.0022 \\
\hline Discharge & $509(90.2)$ & 441 (92.1) & $68(80.0)$ & \\
\hline Death & $55(9.8)$ & $38(7.9)$ & $17(20.0)$ & \\
\hline
\end{tabular}

ARDS: acute respiratory distress syndrome; DIC: disseminated intravascular coagulation; IQR: interquartile range. 
Table 2. Laboratory results among different groups

\begin{tabular}{|c|c|c|c|c|}
\hline \multirow[b]{2}{*}{ Characteristic } & \multicolumn{4}{|c|}{ Median (IQR) } \\
\hline & $\begin{array}{c}\text { Total } \\
(n=564)\end{array}$ & $\begin{array}{c}\text { Non-diabetes } \\
(n=479)\end{array}$ & $\begin{array}{c}\text { Diabetes } \\
(\mathrm{n}=\mathbf{8 5})\end{array}$ & $P$ value \\
\hline \multicolumn{5}{|l|}{ Blood cell count } \\
\hline White blood cell count, $\times 10^{9} / \mathrm{L}$ (normal range $3.5-9.5$ ) & $5.02(3.94-6.93)$ & $4.97(3.90-6.81)$ & $5.95(4.36-7.47)$ & 0.0781 \\
\hline Neutrophil count, $\times 10^{9} / \mathrm{L}$ (normal range 1.6-6.3) & $3.30(2.38-5.01)$ & $3.17(2.31-4.80)$ & $3.88(3.00-6.01)$ & 0.0020 \\
\hline Lymphocyte count, $\times 10^{9} / \mathrm{L}$ (normal range 1.1-3.2) & $1.02(0.65-1.50)$ & $1.05(0.67-1.53)$ & $0.82(0.60-1.34)$ & 0.0153 \\
\hline Monocyte count, $\times 10^{9} / \mathrm{L}$ (normal range $0.1-0.6$ ) & $0.39(0.29-0.51)$ & $0.39(0.29-0.51)$ & $0.36(0.25-0.52)$ & 0.1179 \\
\hline Platelet count, $\times 10^{9} / \mathrm{L}$ (normal range 125-350) & $190.0(141.5-244.5)$ & $193.0(142.0-246.5)$ & $181.0(128.0-233.5)$ & 0.1463 \\
\hline \multicolumn{5}{|l|}{ Blood lipids and electrolytes } \\
\hline Total Cholesterol, mmol/L (normal range 2.8-5.2) & $3.74(3.12-4.36)$ & $3.89(3.00-4.32)$ & $3.38(2.87-4.35)$ & 0.0206 \\
\hline Triglyceride, mmol/L (normal range 0.56-1.7) & $0.95(0.71-1.39)$ & $0.93(0.70-1.38)$ & $1.05(0.76-1.69)$ & 0.1098 \\
\hline $\mathrm{HDL}$, mmol/L (normal range 0.9-2.1) & $1.13(0.95-1.36)$ & $1.14(0.97-1.32)$ & $1.08(0.84-1.24)$ & 0.0055 \\
\hline LDL, mmol/L (normal range 1-3.35) & $2.12(1.70-2.63)$ & $2.15(1.73-2.65)$ & $1.92(1.57-2.56)$ & 0.0364 \\
\hline sdLDL, mmol/L (normal range 95-538) & $137.5(95.8-213.0)$ & $138.0(94.8-213.0)$ & $134.5(97.0-215.3)$ & 0.9037 \\
\hline \multicolumn{5}{|l|}{ Serum } \\
\hline Potassium, mmol/L (normal range 3.5-5.3) & $3.82(3.46-4.18)$ & $3.80(3.46-4.15)$ & $3.89(3.53-4.45)$ & 0.0997 \\
\hline Calcium, mmol/L (normal range 2.11-2.52) & $2.19(2.09-2.31)$ & $2.19(2.10-2.31)$ & $2.16(2.00-2.29)$ & 0.0589 \\
\hline \multicolumn{5}{|l|}{ Inflammatory biomarkers } \\
\hline hsCRP, mg/L (normal range 0-3) & $20.30(2.50-58.59)$ & $18.0(2.15-55.0)$ & $34.6(6.68-81.73)$ & 0.0045 \\
\hline Procalcitonin, ng/mL (normal range 0-0.1) & $0.06(0.04-0.17)$ & $0.06(0.04-0.16)$ & $0.09(0.05-0.26)$ & 0.0941 \\
\hline SAA, mg/L (normal range $0-10$ ) & $74.9(12.0-173.1)$ & $87.6(12.0-206.5)$ & $34.7(10.2-90.92)$ & 0.1308 \\
\hline \multicolumn{5}{|l|}{ Cardiac biomarkers } \\
\hline TnT, ng/mL (normal range 0-0.014) & $0.009(0.006-0.0140)$ & $0.008(0.006-0.013)$ & $0.013(0.009-0.032)$ & $<0.0001$ \\
\hline Creatine kinase-MB, ng/mL (normal range 0-6.22) & $1.16(1.00-2.49)$ & $1.19(0.76-2.22)$ & $1.73(0.97-3.51)$ & 0.0050 \\
\hline Myoglobulin, ng/mL (normal range 7.4-105.7) & $43.1(27.2-78.4)$ & $42.5(26.2-76.1)$ & $49.6(27.2-146.5)$ & 0.0121 \\
\hline NT-proBNP, pg/mL (normal range 0-222) & $157.5(50.2-438.5)$ & $134.9(48.6-422.9)$ & $271.9(99.8-618.0)$ & 0.0037 \\
\hline \multicolumn{5}{|l|}{ Blood gas analysis } \\
\hline $\mathrm{PaO}_{2}, \mathrm{mmHg}$ (normal range 70-107) & $87.0(66.0-118.5)$ & $92.0(69.0-120.5)$ & $74.5(55.3-106.3)$ & 0.0122 \\
\hline $\mathrm{PaO}_{2} / \mathrm{FiO}_{2}, \mathrm{mmHg}$ & $376.0(232.0-481.0)$ & $385.5(248.0-491.3)$ & $290.5(163.6-400.0)$ & 0.0014 \\
\hline $\mathrm{PaCO}_{2}, \mathrm{mmHg}$ (normal range 35-45) & $39.0(34.0-45.0)$ & $40.0(34.0-45.0)$ & $36.0(31.5-45.5)$ & 0.2094 \\
\hline $\mathrm{PH}$ (normal range 7.35-7.45) & $7.42(7.39-7.45)$ & $7.42(7.39-7.45)$ & $7.42(7.38-7.45)$ & 0.5954 \\
\hline $\mathrm{BE}, \mathrm{mmol} / \mathrm{L}$ (normal range $-3-3$ ) & $1.40(-0.50-3.1)$ & $1.50(-0.10-3.10)$ & $0.50(-2.55-2.90)$ & 0.0179 \\
\hline \multicolumn{5}{|l|}{ Liver and renal function } \\
\hline Alanine Aminotransferase, IU/L (normal range 9-50) & $23.0(15.0-36.0)$ & $23.0(15.0-35.9)$ & $24.0(15.0-38.5)$ & 0.5668 \\
\hline Aspartate aminotransferase, IU/L (normal range 15-40) & $26.0(18.0-37.0)$ & $25.5(18.0-37.0)$ & $26.5(17.8-38.3)$ & 0.8051 \\
\hline Total protein, g/L (normal range 65-85) & $64.1(60.3-67.8)$ & $64.1(60.5-67.7)$ & $63.7(59.3-68.5)$ & 0.6828 \\
\hline Albumin, g/L (normal range 40-55) & $37.4(33.1-40.8)$ & $37.6(33.5-41.0)$ & 34.8 (30.9-39.3) & 0.0055 \\
\hline Globulin, g/L (normal range 20-40) & $26.5(23.9-30.0)$ & $26.4(23.7-29.7)$ & $27.9(25.8-32.5)$ & 0.0031 \\
\hline Total bilirubin, $\mu \mathrm{mol} / \mathrm{L}$ (normal range 2-23) & $7.9(5.8-11.0)$ & $7.9(5.7-10.8)$ & $9.2(7.2-12.7)$ & 0.0051 \\
\hline Direct bilirubin, $\mu \mathrm{mol} / \mathrm{L}$ (normal range $0-8$ ) & $3.0(2.0-4.1)$ & $3.0(2.0-4.0)$ & $3.8(2.2-5.6)$ & 0.0034 \\
\hline Creatinine, $\mu \mathrm{mol} / \mathrm{L}$ (normal range 57-97) & $63.0(53.0-74.3)$ & $63.0(53.0-73.0)$ & $66.0(54.0-79.0)$ & 0.3139 \\
\hline Urea nitrogen, $\mu \mathrm{mol} / \mathrm{L}$ (normal range 3.1-8) & $4.32(3.40-5.81)$ & $4.27(3.36-5.56)$ & $4.84(3.63-6.47)$ & 0.0183 \\
\hline
\end{tabular}

HDL: high-density lipoprotein; sdLDL: small dense low-density lipoprotein; LDL: low-density lipoprotein; CRP: C-reactive protein; SAA: serum amyloid A; TnT: troponin T; CK-MB: creatine kinase-MB; NT-proBNP: N-terminal pro-brain natriuretic peptide. 
[1.57-2.56] vs. $2.15[1.73-2.65] \mathrm{mmol} / \mathrm{L} ; p=0.0364)$. Moreover, patients with diabetes had evidence of more severe respiratory dysfunction, with lower partial pressure of oxygen $\left(\mathrm{PaO}_{2}\right)$ (median [IQR], 74.5 [55.3106.3] vs. 92.0 [69.0-120.5] $\mathrm{mmHg} ; p=0.0122)$, and $\mathrm{PaO}_{2}$ /fraction of inspired oxygen $\left(\mathrm{FiO}_{2}\right)$ (median [IQR], 290.5 [163.3-400.0] vs. 385.5 [248.0-491.3] $\mathrm{mmHg} ; p=0.0014)$. Furthermore, patients with diabetes also had higher levels of urea nitrogen (median [IQR], 4.84 [3.63-6.47] vs. 4.27 [3.36-5.56] $\mu \mathrm{mol} / \mathrm{L}$; $p=0.0183)$. Patients with diabetes also presented higher level of total bilirubin (median [IQR], 9.2 [7.212.7] vs. 7.9 [5.7-10.8] $\mu \mathrm{mol} / \mathrm{L} ; p=0.005 \mathrm{l})$, direct bilirubin (median [IQR], 3.8 [2.2-5.6] vs. 3.0 [2.0$4.0] \mu \mathrm{mol} / \mathrm{L} ; p=0.0034$ ), and lower albumin levels (median [IQR], 34.8 [30.9-39.3] vs. 37.6 [33.5-41.0] $\mathrm{g} / \mathrm{L} ; p=0055)$.

It is worth noting that patients with diabetes presented abnormal levels of multiple indicators of heart function and inflammation. Inflammatory biomarkers, including high-sensitivity CRP (median [IQR], 34.6 [6.68-81.73] vs. 18.0 [2.15-55.0] $\mathrm{mg} / \mathrm{L} ; p=0.0045$ ) and globulin (median [IQR], 27.9 [25.8-32.5] vs. 26.4 [23.7-29.7] g/L; $p<0.0001)$ were significantly higher in patients with diabetes. Patients with diabetes also had significantly higher levels of cardiac injury biomarkers, including troponin $\mathrm{T}$ (TnT) (median [IQR],
$0.013[0.009-0.032]$ vs. $0.008[0.006-0.013] \mathrm{ng} / \mathrm{mL}$; $p<0.0001)$, creatine kinase-myocardial band (CK-MB) test (median [IQR], 1.73 [0.97-3.51] vs. 1.19 [0.76$2.22] \mathrm{ng} / \mathrm{mL} ; p=0.0050$ ), myoglobin (median [IQR], 49.6 [27.2-146.5] vs. 42.5 [26.2-76.1] ng/mL; $p=$ 0.0121 ), and $\mathrm{N}$-terminal pro-brain natriuretic peptide (NT-proBNP) (median [IQR], 271.9 [99.8-618.0] vs. 134.9 [48.6-422.9] pg/mL; $p=0.0037)$.

We further analyzed the dynamic changes in TnT and CRP levels during hospitalization among these patient cohorts (Figure 2). As shown in Figure 2A, the TnT level of patients with diabetes increased significantly during the course of hospitalization compared to that in patients without diabetes (median [IQR], 0.013 [0.007-0.044] vs. 0.010 [0.006-0.019] $\mathrm{ng} / \mathrm{mL}, p=0.0167$ at hospitalization and 0.030 [0.008-0.111] vs. $0.010[0.005-0.0203] \mathrm{ng} / \mathrm{mL}$, $p=0.0040$ approaching discharge). Both groups of patients exhibited high CRP levels during the course of hospitalization (median [IQR], 19.1 [4.4-104.5] vs. $11.6[2.6-49.2] \mathrm{mg} / \mathrm{L}, p=0.1042$ at hospitalization). The CRP levels of patients with diabetes were controlled close to the normal range (median [IQR], $5.7[1.35-30.6] \mathrm{mg} / \mathrm{L}$ ), with no significant difference compared to patients without diabetes (median [IQR], $3.5[1.4-18.6] \mathrm{mg} / \mathrm{L})$ when approaching discharge (Figure 2B).
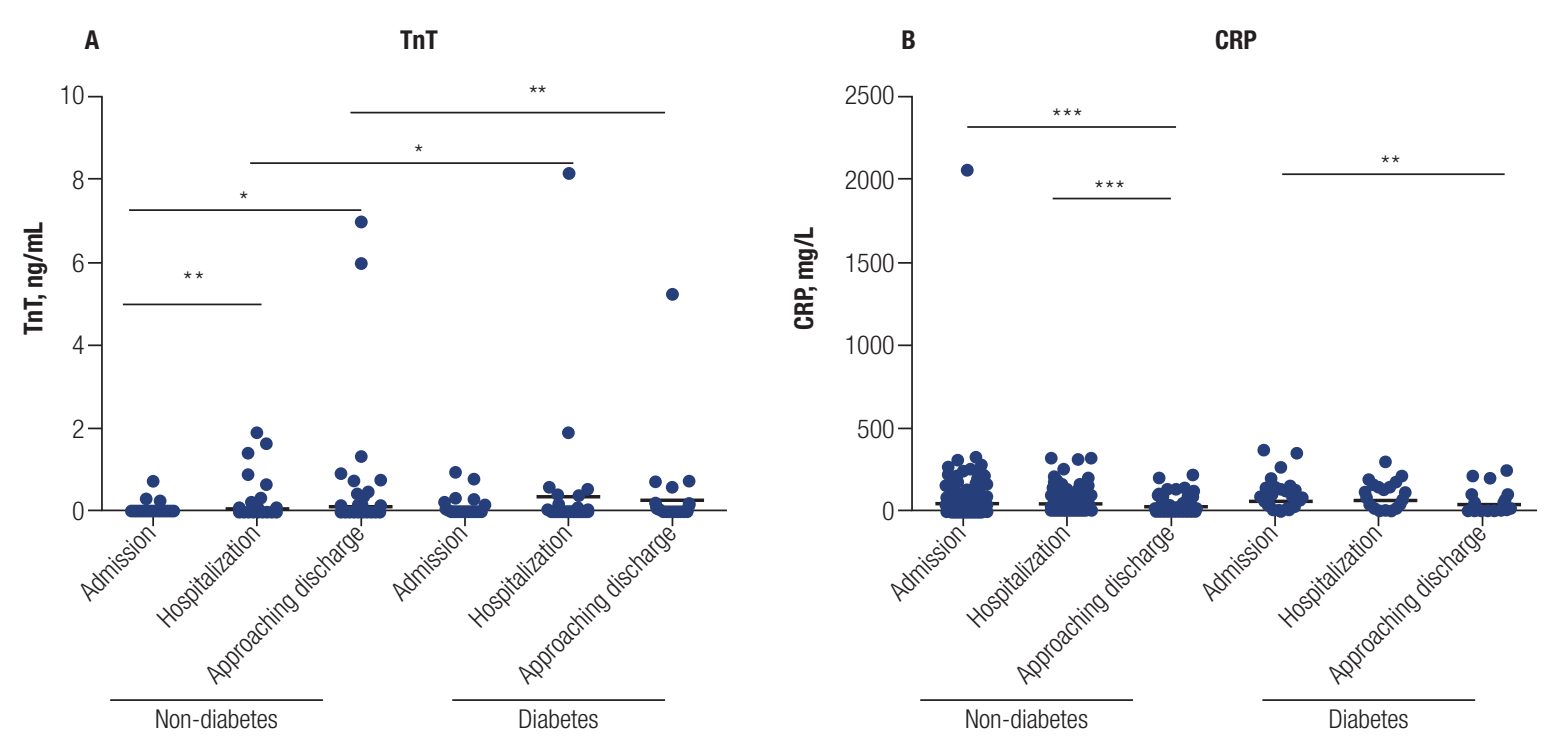

Figure 2. Dynamic change of TnT, and CRP during hospitalization.

A.TnT; B. CRP. The data were expressed as the median and IQR. Mann-Whitney U test was used $\left({ }^{\star} p<0.05,{ }^{\star *} p<0.01,{ }^{* \star \star} p<0.001\right)$. 


\section{Treatments, complications, and clinical outcomes}

The median time from symptom onset to admission was 10 (IQR, 7-15) days in patients with diabetes, similar to that in patients without diabetes $(p=0.7271$; Table 1). There was also no significant difference in hospitalization time between the two groups. During hospitalization, patients with diabetes more frequently developed complications, including ARDS (20 [23.5\%] vs. $41[8.6 \%] ; p=0.0002)$, acute heart failure (10 [11.8\%] vs. $15[3.1 \%] ; p=0.0017)$, metabolic acidosis $(8[9.4 \%]$ vs. $11[2.3 \%] ; p=0.0036)$, acute kidney injury $(8[9.4 \%]$ vs. $19[4.0 \%] ; p=0.0477)$, and disseminated intravascular coagulation (DIC) $(4[4.7 \%]$ vs. $3[0.6 \%]$; $p=0.0118$ ) compared to patients without diabetes (Table 1). There were no significant differences in the incidence of bacterial infection and acute liver injury between the two groups.

A total of 355 patients (62.9\%) underwent respiratory support, with 305 (54.1\%), 35 (6.2\%), and $15(2.7 \%)$ receiving nasal cannula, non-invasive ventilation, and invasive mechanical ventilation, respectively. Most patients received antiviral (351 [62.2\%] and antibacterial (407 [72.1\%]) therapies during hospitalization. Chinese medicine, glucocorticoids, and immunoglobulins were administered to $343(60.8 \%), 193(34.2 \%)$, and 90 (16.0\%) patients, respectively. Overall, the rates of these treatments did not differ significantly between patients with and without diabetes.

The relationship between diabetes and death was the focus of the present study. We found that diabetes was associated with a nearly 3.0 -fold and significantly enhanced risk of death with COVID-19 (odds ratio [OR]: 2.950 [95\% confidence interval (CI): 1.593$5.463], p<0.001), 0.001)$, which corresponded to an E-value of 5.348. The observed OR of 2.950 could be explained away by an unmeasured confounder that was associated with both diabetes and death by an odds ratio of 5.3-fold each, but weaker confounding could not do so. We excluded 29 patients (four with diabetes and 25 without) transferred to the superior hospital and selected confounders based on their associations with the outcomes of patients or a change in the effect estimate of at least $10 \%$ (Table 3). Multivariate logistic regression analysis indicated that the association between diabetes and outcome of COVID-19 did not change markedly after adjusting for hypertension, liver disease, and kidney disease (OR: 2.195 [95\%CI: 1.048$4.599], p=0.037$, which produces E-value $=3.815$ for
Table 3. Associations of covariates with the outcome of patients with COVID-19 $(\mathrm{N}=535)^{1}$

\begin{tabular}{lc}
\hline & $\begin{array}{c}\text { Status } \\
\text { (0: survival; 1: dead) }\end{array}$ \\
\hline Age, yr & $0.099^{*}(0.070-0.127)$ \\
Hypertension & \\
Yes & $0.878^{*}(0.328-1.430)$ \\
No & Reference \\
liver disease & \\
Yes & $1.343^{*}(0.467-2.220)$ \\
No & Reference \\
Kidney disease & \\
Yes & $1.924^{*}(1.060-2.788)$ \\
No & Reference \\
\hline
\end{tabular}

${ }^{1}$ Values are regression coefficients (95\% Confidence Interval) from univariate regression models and reflect differences in patients' outcomes per unit change of each covariate and for different categories of each covariate as compared to the reference group. ${ }^{*} P$ value $<0.05$.

the estimate. After adjusting for selected controlled confounders, the observed odds ratio of 2.195 could be explained away by an unmeasured confounder that was associated with both diabetes and death by an odds ratio of 3.8 -fold each, above and beyond the measured confounders, but weaker confounding could not do so. Based on these 535 patients, we constructed a Kaplan-Meier survival curve. The results showed shorter durations from admission to 40-day follow-up in patients with diabetes compared to those without diabetes after giving a landmark of $40($ Figure $3 \mathrm{~A}$, mean $=31.621, \mathrm{SE}=1.704 ;$ mean $=$ $36.008, \mathrm{SE}=0.633 ; p=0.0012)$. The corresponding hazard function shown in Figure $3 \mathrm{~B}$ indicates a higher probability of death in patients with diabetes than in patients without diabetes.

\section{Characteristics of COVID-19 patients with diabetes with in-hospital death}

As diabetes is an independent risk factor related to in-hospital death or poor prognosis in patients with COVID-19, we further analyzed the clinical characteristics of survivors and non-survivors of COVID-19. Among 85 COVID-19 patients with diabetes, those with older age and men were more likely to die. Compared to non-survivors with diabetes, survivors had longer hospitalization times (10 [4-13] vs. 19.5 [12-26] days, $p<0.0001)$. More non-survivors reported comorbidities related to cardiovascular disease $(9,52.9 \%)$ and malignancy (4, 23.5\%). Non-survivors received fewer Chinese 

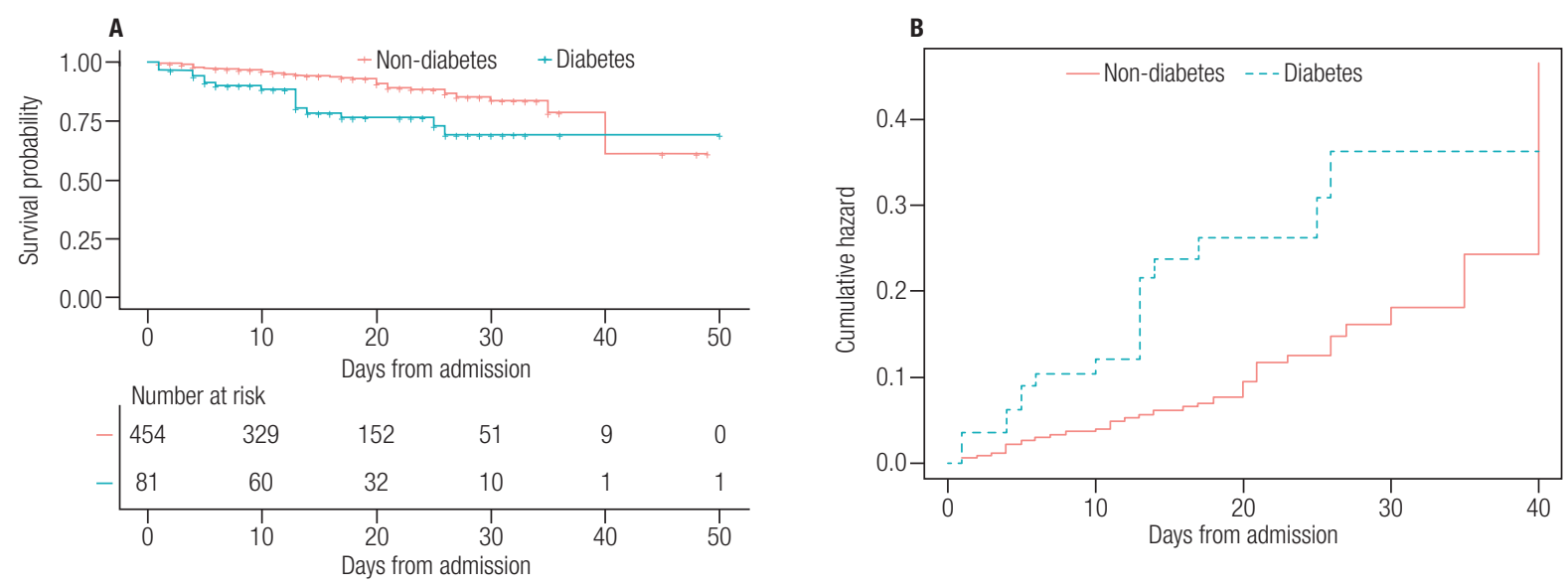

Figure 3. Kaplan-Meier plots of survival probability in hospitalized patients with COVID-19. A. Kaplan-Meier survival curves for mortality during the time from admission. Mortality was significantly higher in patients with diabetes. B. The hazard Function during the time from admission to 40-day follow up.

medicine $(6[35.3 \%]$ vs. $48[70.6 \%], p=0.0107)$, and more glucocorticoid treatment $(12[70.5 \%]$ vs. 22 $[32.3 \%], p=0.0057)$. Moreover, more non-survivors received mechanical ventilation. All these baseline characteristics interplayed the rapid progress of the disease in the COVID-19 patients with diabetes with in-hospital death (Table 4).

Compared to survivors, non-survivors presented lower lymphocyte (median [IQR], 0.53 [0.21$0.82]$ vs. $\left.0.99[0.65-1.48] \times 10^{9} / \mathrm{L} ; p=0.0005\right)$ and monocyte (median [IQR], 0.26 [0.15-0.34] vs. 0.42 $\left.[0.28-0.52] \times 10^{9} / \mathrm{L} ; p=0.0005\right)$ counts and higher levels of hsCRP (median [IQR], 86.7 [46.3-123.7] vs. $22.5[4.3-64.2] \mathrm{mg} / \mathrm{L} ; p=0.0002)$, procalcitonin (median [IQR], $0.227[0.117-0.613]$ vs. 0.059 [0.039-0.121] ng/mL; $p=0.016)$, and SAA (median [IQR], 90.92 [79.51-180.8] vs. 22.5 l [8.71-62.91] $\mathrm{mg} / \mathrm{L} ; p=0.008)$, which indicated that non-surviving patients with diabetes tended to present stronger inflammatory responses. Moreover, non-survivors with diabetes had lower $\mathrm{PaO}_{2}$ (median [IQR], 50.0 [39.5-61.3] vs. 81.0 [65.5-108.5] $\mathrm{mmHg} ; p$ $=0.0004$ ) and $\mathrm{PaO}_{2} / \mathrm{FiO}_{2}$ (median [IQR], 131.0 [100.0-261.9] vs. 350.0 [234.5-431.0] $\mathrm{mmHg}$; $p=0.0004)$, which indicated that non-survivors developed more severe respiratory dysfunction. In addition, non-survivors with diabetes had multiple abnormal laboratory values related to cardiac, hepatic, and renal impairment, including higher levels of TnT, CK-MB, myoglobulin, NT-proBNP, aspartate aminotransferase, and urea nitrogen and lower levels of albumin (Table 5 ).

\section{DISCUSSION}

According to World Health Organization statistics, the number of globally confirmed cases reached $13,150,645$ with 574,464 deaths in 216 countries by July 15, 2020 (15). Early studies indicated that diabetes was one of the most prevalent comorbidities in patients with COVID-19 $(3,16)$. The present cohort study provided detailed clinical characteristics and risk factors associated with clinical outcomes in patients with COVID-19 with and without diabetes admitted to the No.7 Hospital of Wuhan between January 20 and March 15, 2020. The overall case fatality rate in mainland China was 5.4\% (4,649 deaths out of 85,677 confirmed cases as of July 15,2020$)$ (15). In our study, the prevalence of diabetes in COVID-19 patients was $15.1 \%$, consistent with previous reports of proportions of COVID-19 patients with diabetes ranging from $2.7 \%$ to $24.9 \%$ (17-19). The in-hospital mortality rate in patients with diabetes was markedly higher than that in patients without diabetes $(20.0 \%$ vs. $7.9 \%, p=0.0022)$, in line with previous findings. Our data demonstrated that diabetes can be considered a risk factor for death in patients with COVID-19 in Wuhan. Previous studies on the SARS pandemic indicated that diabetes, as well as fasting blood glucose (FBG) level, were associated with severe or lethal SARS-CoV infections (20). Diabetes was also reported to be a significant risk factor for both mortality and morbidity due to MERS-CoV $(21,22)$. The presence of diabetes was also associated with worse clinical outcomes in patients infected with the HINl influenza A virus $(17,23)$. Combined 
Table 4. The clinical characteristics and laboratory values of survivors and non-survivors in COVID-19 patients with diabetes

\begin{tabular}{|c|c|c|c|}
\hline Characteristic & $\begin{array}{l}\text { Survivors } \\
(\mathrm{n}=68)\end{array}$ & $\begin{array}{l}\text { Non-survivors } \\
\quad(\mathrm{n}=17)\end{array}$ & $P$ value \\
\hline Age-median (range) & $62.5(34-89)$ & $73(58-91)$ & 0.0002 \\
\hline Male & $36(52.9)$ & $13(76.5)$ & 0.1026 \\
\hline Hospitalization, median (IQR), d & $19.5(12-26)$ & $10(4-13)$ & $<0.0001$ \\
\hline \multicolumn{4}{|l|}{ Comorbidity - No. (\%) } \\
\hline Cardiovascular disease & $16(23.5)$ & $9(52.9)$ & 0.034 \\
\hline Cerebrovascular disease & $1(1.5)$ & $2(22.8)$ & 0.1005 \\
\hline Hypertension & 45 (66.2) & $13(76.5)$ & 0.5635 \\
\hline Malignancy & $1(1.5)$ & $4(23.5)$ & 0.0051 \\
\hline Kidney disease & $2(2.9)$ & $3(17.6)$ & 0.0523 \\
\hline \multicolumn{4}{|l|}{ Medical control for diabetes } \\
\hline No medication & $28(41.2)$ & $8(47.1)$ & 0.7852 \\
\hline Oral medication & $37(54.4)$ & $3(17.6)$ & 0.0073 \\
\hline Insulin & $21(30.6)$ & $8(47.1)$ & 0.2563 \\
\hline \multicolumn{4}{|l|}{ Clinical treatment } \\
\hline Antiviral treatment & 43 (63.2) & $10(58.8)$ & 0.7837 \\
\hline Antibiotics & $49(72.1)$ & $13(76.5)$ & 1.0000 \\
\hline Chinese Medicine & $48(70.6)$ & $6(35.3)$ & 0.0107 \\
\hline Glucocorticoid & $22(32.3)$ & $12(70.5)$ & 0.0057 \\
\hline Immune globulin & $13(19.1)$ & $7(41.1)$ & 0.1056 \\
\hline \multicolumn{4}{|l|}{ Respiratory support } \\
\hline Nasal cannula & $42(61.8)$ & $6(35.3)$ & 0.0005 \\
\hline Non-invasive ventilation & $3(4.4)$ & $7(41.2)$ & \\
\hline Invasive ventilation & $1(1.5)$ & $4(23.5)$ & \\
\hline \multicolumn{4}{|l|}{ Laboratory findings on admission } \\
\hline FBS (fasting blood glucose), mmol/L (normal range 3.9-5.8) & $7.98(6.08-12.62)$ & $9.45(6.53-14.20)$ & 0.4692 \\
\hline Arterial blood sugar, mmol/L (normal range 3.9-7.8) & $9.30(6.20-13.40)$ & $10.65(6.65-14.90)$ & 0.3751 \\
\hline White blood cell count, $\times 10^{9} / \mathrm{L}$ (normal range 3.5-9.5) & $5.68(4.37-7.54)$ & $6.12(3.60-7.99)$ & 0.8372 \\
\hline Neutrophil count, $\times 10^{9} / \mathrm{L}$ (normal range 1.6-6.3) & $3.86(2.99-5.97)$ & $5.11(3.05-7.18)$ & 0.4207 \\
\hline Lymphocyte count, $\times 10^{9} / L$ (normal range 1.1-3.2) & $0.99(0.65-1.48)$ & $0.53(0.21-0.82)$ & 0.0005 \\
\hline Monocyte count, $\times 10^{9} / \mathrm{L}$ (normal range $0.1-0.6$ ) & $0.42(0.28-0.52)$ & $0.26(0.15-0.34)$ & 0.0066 \\
\hline Platelet count, $\times 10^{9} / \mathrm{L}$ (normal range $125-350$ ) & $189.0(135.5-251.0)$ & $114.0(82.0-152.5)$ & 0.0003 \\
\hline \multicolumn{4}{|l|}{ Blood lipids and electrolytes } \\
\hline Total cholesterol, mmol/L (normal range 2.8-5.2) & $3.48(2.86-4.42)$ & $3.31(2.3-3.86)$ & 0.2283 \\
\hline Triglyceride, mmol/L (normal range 0.56-1.7) & $1.01(0.67-1.71)$ & $1.19(1.03-1.54)$ & 0.1145 \\
\hline $\mathrm{HDL}$, mmol/L (normal range 0.9-2.1) & $1.10(0.85-1.25)$ & $0.99(0.81-1.24)$ & 0.5957 \\
\hline LDL, mmol/L (normal range 1-3.35) & $2.07(1.63-2.60)$ & $1.60(1.05-2.06)$ & 0.0459 \\
\hline sdLDL, mmol/L (normal range 95-538) & $146.0(101.3-244.8)$ & $97.0(65.0-151.0)$ & 0.0127 \\
\hline Potassium, mmol/L (normal range 3.5-5.3) & $3.89(3.57-4.33)$ & $4.00(3.11-4.89)$ & 0.8854 \\
\hline Calcium, mmol/L (normal range 2.11-2.52) & $2.18(2.02-2.31)$ & $2.04(1.98-2.18)$ & 0.0748 \\
\hline hsCRP, mg/L (normal range 0-3) & $22.5(4.3-64.2)$ & $86.7(46.3-123.7)$ & 0.0002 \\
\hline Procalcitonin, $\mathrm{ng} / \mathrm{mL}$ (normal range $0-0.1$ ) & $0.059(0.039-0.121)$ & $0.227(0.117-0.613)$ & 0.016 \\
\hline SAA, mg/L (normal range $0-10$ ) & $22.51(8.71-62.91)$ & 90.92 (79.51-180.8) & 0.008 \\
\hline TnT, ng/mL (normal range 0-0.014) & $0.010(0.008-0.018)$ & $0.104(0.027-0.275)$ & $<0.0001$ \\
\hline Creatine kinase-MB, ng/mL (normal range 0-6.22) & $1.49(0.78-2.87)$ & $3.73(1.48-16.22)$ & 0.0095 \\
\hline
\end{tabular}




\begin{tabular}{|c|c|c|c|}
\hline Characteristic & $\begin{array}{c}\text { Survivors } \\
(n=68)\end{array}$ & $\begin{array}{c}\text { Non-survivors } \\
(n=17)\end{array}$ & $P$ value \\
\hline Myoglobulin, ng/mL (normal range 7.4-105.7) & $39.8(28.7-83.1)$ & $145.9(72.8-191.8)$ & 0.0023 \\
\hline NT-proBNP, pg/mL (normal range 0-222) & $255.2(93.9-399.5)$ & 778.5 (343.6-9691) & 0.0015 \\
\hline $\mathrm{PaO}_{2}, \mathrm{mmHg}$ (normal range 70-107) & $81.0(65.5-108.5)$ & $50.0(39.5-61.3)$ & 0.0004 \\
\hline $\mathrm{PaO}_{2} / \mathrm{FiO}_{2}, \mathrm{mmHg}$ & $350.0(234.5-431.0)$ & $131.0(100.0-261.9)$ & 0.0004 \\
\hline $\mathrm{PaCO}_{2}, \mathrm{mmHg}$ (normal range $35-45$ ) & $39.0(35.0-46.3)$ & $31.0(23.5-38.0)$ & 0.0125 \\
\hline $\mathrm{PH}$ (normal range 7.35-7.45) & $7.41(7.31-7.45)$ & $7.41(7.00-7.48)$ & 0.9231 \\
\hline $\mathrm{BE}, \mathrm{mmol} / \mathrm{L}$ (normal range $-3-3$ ) & $1.40(-0.9-3.13)$ & $-3.65(-5.28-0.18)$ & 0.0001 \\
\hline Alanine aminotransferase, IU/L (normal range 9-50) & $22.0(13.3-35.8)$ & $27.0(25.5-46.0)$ & 0.0881 \\
\hline Aspartate aminotransferase, IU/L (normal range 15-40) & $26.0(17.0-37.5)$ & $35.0(25.0-68.0)$ & 0.0407 \\
\hline Total protein, g/L (normal range 65-85) & $63.6(59.6-69.9)$ & $63.8(57.5-65.1)$ & 0.2261 \\
\hline Albumin, g/L (normal range 40-55) & $36.3(30.9-41.2)$ & $32.5(30.0-35.0)$ & 0.0246 \\
\hline Globulin, g/L (normal range 20-40) & $27.6(25.9-32.2)$ & $29.0(24.4-35.3)$ & 0.5273 \\
\hline Total bilirubin, $\mu \mathrm{mol} / \mathrm{L}$ (normal range 2-23) & $9.2(7.2-12.4)$ & $9.5(7.5-19.88)$ & 0.4404 \\
\hline Direct bilirubin, $\mu \mathrm{mol} / \mathrm{L}$ (normal range $0-8$ ) & $3.45(2.2-5.33)$ & $4.7(3.2-8.8)$ & 0.0512 \\
\hline Creatinine, $\mu \mathrm{mol} / \mathrm{L}$ (normal range 57-97) & $66.0(54.0-79.0)$ & $68.0(50.0-112.0)$ & 0.8287 \\
\hline Urea nitrogen, $\mu \mathrm{mol} / \mathrm{L}$ (normal range 3.1-8) & $4.71(3.58-6.16)$ & $6.95(3.72-12.28)$ & 0.0256 \\
\hline
\end{tabular}

HDL: high-density lipoprotein; sdLDL: small dense low-density lipoprotein; LDL: low-density lipoprotein; CRP: C-reactive protein; SAA: serum amyloid A; TnT: troponin T; CK-MB: creatine kinase-MB; NT-proBNP: N-terminal pro-brain natriuretic peptide.

Table 5. The laboratory values of survivors and non-survivors in COVID-19 patients with diabetes

\begin{tabular}{|c|c|c|c|}
\hline \multirow{2}{*}{ Characteristic } & \multicolumn{3}{|c|}{ Median (IQR) } \\
\hline & Survivors $(n=68)$ & Non-survivors $(n=17)$ & $P$ value \\
\hline FBS (fasting blood glucose), mmol/L (normal range 3.9-5.8) & $7.98(6.08-12.62)$ & $9.45(6.53-14.20)$ & 0.4692 \\
\hline Arterial blood sugar, mmol/L (normal range 3.9-7.8) & $9.30(6.20-13.40)$ & $10.65(6.65-14.90)$ & 0.3751 \\
\hline \multicolumn{4}{|l|}{ Blood cell count } \\
\hline White blood cell count, $\times 10^{9} / \mathrm{L}$ (normal range 3.5-9.5) & $5.68(4.37-7.54)$ & $6.12(3.60-7.99)$ & 0.8372 \\
\hline Neutrophil count, $\times 10^{9} / \mathrm{L}$ (normal range 1.6-6.3) & $3.86(2.99-5.97)$ & $5.11(3.05-7.18)$ & 0.4207 \\
\hline Lymphocyte count, $\times 10^{9} / \mathrm{L}$ (normal range 1.1-3.2) & $0.99(0.65-1.48)$ & $0.53(0.21-0.82)$ & 0.0005 \\
\hline Monocyte count, $\times 10^{9} / \mathrm{L}$ (normal range $0.1-0.6$ ) & $0.42(0.28-0.52)$ & $0.26(0.15-0.34)$ & 0.0066 \\
\hline Platelet count, $\times 10 \% / L$ (normal range 125-350) & $189.0(135.5-251.0)$ & $114.0(82.0-152.5)$ & 0.0003 \\
\hline \multicolumn{4}{|l|}{ Blood lipids and electrolytes } \\
\hline Total cholesterol, mmol/L (normal range 2.8-5.2) & $3.48(2.86-4.42)$ & $3.31(2.3-3.86)$ & 0.2283 \\
\hline Triglyceride, mmol/L (normal range 0.56-1.7) & $1.01(0.67-1.71)$ & $1.19(1.03-1.54)$ & 0.1145 \\
\hline $\mathrm{HDL}$, mmol/L (normal range 0.9-2.1) & $1.10(0.85-1.25)$ & $0.99(0.81-1.24)$ & 0.5957 \\
\hline LDL, mmol/L (normal range 1-3.35) & $2.07(1.63-2.60)$ & $1.60(1.05-2.06)$ & 0.0459 \\
\hline sdLDL, mmol/L (normal range 95-538) & $146.0(101.3-244.8)$ & $97.0(65.0-151.0)$ & 0.0127 \\
\hline \multicolumn{4}{|l|}{ Serum } \\
\hline Potassium, mmol/L (normal range 3.5-5.3) & $3.89(3.57-4.33)$ & $4.00(3.11-4.89)$ & 0.8854 \\
\hline Calcium, mmol/L (normal range 2.11-2.52) & $2.18(2.02-2.31)$ & $2.04(1.98-2.18)$ & 0.0748 \\
\hline \multicolumn{4}{|l|}{ Inflammatory biomarkers } \\
\hline hsCRP, mg/L (normal range $0-3$ ) & $22.5(4.3-64.2)$ & $86.7(46.3-123.7)$ & 0.0002 \\
\hline Procalcitonin, ng/mL (normal range 0-0.1) & $0.059(0.039-0.121)$ & $0.227(0.117-0.613)$ & 0.016 \\
\hline SAA, mg/L (normal range 0-10) & $22.51(8.71-62.91)$ & $90.92(79.51-180.8)$ & 0.008 \\
\hline \multicolumn{4}{|l|}{ Cardiac biomarkers } \\
\hline TnT, ng/mL (normal range 0-0.014) & $0.010(0.008-0.018)$ & $0.104(0.027-0.275)$ & $<0.0001$ \\
\hline Creatine kinase-MB, ng/mL (normal range 0-6.22) & $1.49(0.78-2.87)$ & $3.73(1.48-16.22)$ & 0.0095 \\
\hline
\end{tabular}




\begin{tabular}{|c|c|c|c|}
\hline \multirow{2}{*}{ Characteristic } & \multicolumn{3}{|c|}{ Median (IQR) } \\
\hline & Survivors $(n=68)$ & Non-survivors $(n=17)$ & $P$ value \\
\hline Myoglobulin, ng/mL (normal range 7.4-105.7) & $39.8(28.7-83.1)$ & $145.9(72.8-191.8)$ & 0.0023 \\
\hline NT-proBNP, pg/mL (normal range 0-222) & $255.2(93.9-399.5)$ & $778.5(343.6-9691)$ & 0.0015 \\
\hline \multicolumn{4}{|l|}{ Blood gas analysis } \\
\hline $\mathrm{PaO}_{2}, \mathrm{mmHg}$ (normal range $70-107$ ) & $81.0(65.5-108.5)$ & $50.0(39.5-61.3)$ & 0.0004 \\
\hline $\mathrm{PaO}_{2} / \mathrm{FiO}_{2}, \mathrm{mmHg}$ & $350.0(234.5-431.0)$ & $131.0(100.0-261.9)$ & 0.0004 \\
\hline $\mathrm{PaCO}_{2}, \mathrm{mmHg}$ (normal range 35-45) & $39.0(35.0-46.3)$ & $31.0(23.5-38.0)$ & 0.0125 \\
\hline PH (normal range 7.35-7.45) & $7.41(7.31-7.45)$ & $7.41(7.00-7.48)$ & 0.9231 \\
\hline $\mathrm{BE}, \mathrm{mmol} / \mathrm{L}$ (normal range $-3-3$ ) & $1.40(-0.9-3.13)$ & $-3.65(-5.28-0.18)$ & 0.0001 \\
\hline \multicolumn{4}{|l|}{ Liver and renal function } \\
\hline Alanine aminotransferase, IU/L (normal range 9-50) & $22.0(13.3-35.8)$ & $27.0(25.5-46.0)$ & 0.0881 \\
\hline Aspartate aminotransferase, IU/L (normal range 15-40) & $26.0(17.0-37.5)$ & $35.0(25.0-68.0)$ & 0.0407 \\
\hline Total protein, g/L (normal range 65-85) & $63.6(59.6-69.9)$ & $63.8(57.5-65.1)$ & 0.2261 \\
\hline Albumin, g/L (normal range 40-55) & $36.3(30.9-41.2)$ & $32.5(30.0-35.0)$ & 0.0246 \\
\hline Globulin, g/L (normal range 20-40) & $27.6(25.9-32.2)$ & $29.0(24.4-35.3)$ & 0.5273 \\
\hline Total bilirubin, $\mu \mathrm{mol} / \mathrm{L}$ (normal range 2-23) & $9.2(7.2-12.4)$ & $9.5(7.5-19.88)$ & 0.4404 \\
\hline Direct bilirubin, $\mu \mathrm{mol} / \mathrm{L}$ (normal range $0-8$ ) & $3.45(2.2-5.33)$ & $4.7(3.2-8.8)$ & 0.0512 \\
\hline Creatinine, $\mu \mathrm{mol} / \mathrm{L}$ (normal range 57-97) & $66.0(54.0-79.0)$ & $68.0(50.0-112.0)$ & 0.8287 \\
\hline Urea nitrogen, $\mu \mathrm{mol} / \mathrm{L}$ (normal range 3.1-8) & $4.71(3.58-6.16)$ & $6.95(3.72-12.28)$ & 0.0256 \\
\hline
\end{tabular}

HDL: high-density lipoprotein; sdLDL: small dense low-density lipoprotein; LDL: Iow-density lipoprotein; CRP: C-reactive protein; SAA: serum amyloid A; TnT: troponin T; CK-MB: creatine kinase-MB; NT-proBNP: N-terminal pro-brain natriuretic peptide.

with our data, the cumulative findings confirmed that patients with diabetes are more susceptible to certain infectious diseases.

In this retrospective cohort study, we demonstrated that diabetes was associated with a worse COVID-19 prognosis compared to patients without diabetes with COVID-19. In our study, COVID-19 patients with diabetes were more likely to have comorbidities of hypertension and cardiovascular disease. It is worth noting that diabetic patients presented with significantly higher neutrophil counts and lower lymphocyte counts, indicating that these COVID-19 patients with diabetes exhibited more severe lymphopenia (24). In addition, diabetic patients with COVID-19 presented higher hsCRP levels. These observations suggested that patients with diabetes were prone to more serious infections due to immune system imbalance, which is in with previous findings that patients with COVID-19 had higher levels of cytokines, including interleukin (IL)-2, IL-8, and tumor necrosis factor (TNF)- $\alpha$ (25).

Moreover, diabetic patients with COVID-19 also presented abnormal levels of multiple laboratory findings at hospital admission, including those related to heart (TnT, CK-MB, myoglobulin, and
NT-proBNP), liver (albumin), kidney (urea nitrogen), and lung function $\left(\mathrm{PaO}_{2}, \mathrm{PaO}_{2} / \mathrm{FiO}_{2}\right)$, indicating that SAR-CoV-2 infection may be related to progressive systemic injury in patients with diabetes. Corresponding to these findings, diabetic patients with COVID-19 were more likely to develop more complications, including ARDS, acute heart failure, metabolic acidosis, acute kidney injury, and DIC. Angiotensin-converting enzyme-2 (ACE-2) has been reported as a receptor for both SARS-CoV-2 and SARS-CoV (1). As an enzyme of the renin-angiotensin system (RAS), ACE2 is widely expressed in human tissues, including the lung, kidney, heart, digestive tract, blood vessels, testis, immune cells, and pancreas (26). Therefore, it is unsurprising that COVID-19 patients experience multiple extrapulmonary manifestations and possible complications. Yang and cols. demonstrated that the binding of SARS-CoV to ACE2 in pancreatic islet cells can lead to cell damage and acute diabetes (25). We assume that a similar mechanism related to pancreatic damage may also exist in COVID-19 infection, possibly contributing to hyperglycosemia, worse complications, and mortality, which requires further evidence. 
In our study, $30.9 \%$ of the non-surviving COVID-19 patients had underlying diabetes. Among diabetic patients with COVID-19, there were more male non-survivors. Additionally, non-surviving COVID-19 patients with diabetes exhibited abnormal levels of biomarkers associated with severe forms of lymphopenia; inflammatory response; and cardiac, renal, hepatic, and respiratory system injury, consistent with previous reports $(8,9)$. Combined with dynamic changes during hospitalization, surveillance of the levels of these biomarkers, especially cardiac indicators, may be helpful in the treatment of patients with COVID-19 patients with diabetes.

However, this study has several limitations. First, patients with uncomplicated illness were assigned to Fangcang shelter hospitals as an important component of the national response to the COVID-19 pandemic, which may have resulted in increased enrollment of patients with severe COVID-19. Second, the followup medical data were incomplete, as some cases were transferred. Third, this retrospective study relied on data collected from electronic medical records, from which some information was unavoidably missing.

The results of the present study suggested that diabetes was significantly associated with disease severity and fatal outcomes of COVID-19. Patients with COVID-19 patients with diabetes experienced severe multiple-organ manifestations and complications, especially myocardial and kidney injury, indicating the potential need for more intensive treatment and surveillance in these patients. Long-term observation and prospective study design are needed to assess the effectiveness of treatments specific for COVID-19 patients with diabetes.

Authors' contribution: conception and design of the research: Fu XD, Xiong HR. Acquisition of data: Deng YP, Xie W, Liu T, Wang SY, Meng XB. Analysis and interpretation of the data: Deng YP, Xie W, Wang MR. Statistical analysis: Deng YP, Zan YX, Zheng J. Obtaining financing: None. Writing of the manuscript: Deng YP, Xie W. Critical revision of the manuscript for intellectual content: Fu XD, Xiong HR.

Acknowledgements: we would like to all the medical staff working in No.7 hospital and Zhongnan hospital of Wuhan University for their great effort in taking care of the patients with COVID-19.

Disclosure: no potential conflict of interest relevant to this article was reported

\section{REFERENCES}

1. Cao X. COVID-19: immunopathology and its implications for therapy. Nat Rev Immunol. 2020;20(5):269-70.
2. Jiang $\mathrm{X}$, Rayner S, Luo MH. Does SARS-CoV-2 has a longer incubation period than SARS and MERS? J Med Virol. 2020;92(5):476-8.

3. Guan WJ, Ni ZY, Hu Y, Liang WH, Ou CQ, He JX, et al. Clinical Characteristics of Coronavirus Disease 2019 in China. N Engl J Med. 2020;382(18):1708-20.

4. Tadic M, Cuspidi C, Sala C. COVID-19 and diabetes: Is there enough evidence? J Clin Hypertens (Greenwich). 2020;22(6): 943-8.

5. Shi S, Qin M, CaiY, LiuT, Shen B, Yang F, et al. Characteristics and clinical significance of myocardial injury in patients with severe coronavirus disease 2019. Eur Heart J. 2020;41(22):2070-9.

6. Melmer A, Laimer M. Treatment Goals in Diabetes. Endocr Develop. 2016;31:1-27.

7. Knapp S. Diabetes and infection: is there a link? - A mini-review. Gerontology. 2013;59(2):99-104.

8. Chen Y, Yang D, Cheng B, Chen J, Peng A, Yang C, et al. Clinical Characteristics and Outcomes of Patients with Diabetes and COVID-19 in Association with Glucose-Lowering Medication. Diabetes Care. 2020;43(7):1399-407.

9. Guo W, Li M, Dong Y, Zhou H, Zhang Z, Tian C, et al. Diabetes is a risk factor for the progression and prognosis of COVID-19. Diabetes Metab Res Rev. 2020:e3319.

10. NHC. National Health Commission of the People's Republic of China home page 2020. Available from: http://www.nhc.gov.cn/ yzygj/ggwsylglc/new_list.shtml.

11. Chen S, Zhang Z, Yang J, Wang J, Zhai X, Barnighausen T, et al. Fangcang shelter hospitals: a novel concept for responding to public health emergencies. Lancet. 2020;395(10232):1305-14.

12. Shang $L, X u J, C a o$ B. Fangcang shelter hospitals in COVID-19 pandemic: the practice and its significance. Clin Microbiol Infect. 2020;26(8):976-8.

13. Wang D, Hu B, Hu C, Zhu F, Liu X, Zhang J, et al. Clinical Characteristics of 138 Hospitalized Patients With 2019 Novel Coronavirus-Infected Pneumonia in Wuhan, China. JAMA. 2020;323(11):1061-9.

14. VanderWeele TJ, Ding P. Sensitivity Analysis in Observational Research: Introducing the E-Value. Ann Intern Med. 2017;167(4):268-74.

15. WHO. Coronavirus disease (COVID-19) pandemic: WHO; 2020 [cited 2020]. Available from: https://www.who.int/emergencies/ diseases/novel-coronavirus-2019.

16. Guo T, Fan Y, Chen M, Wu X, Zhang L, He T, et al. Cardiovascular Implications of Fatal Outcomes of Patients with Coronavirus Disease 2019 (COVID-19). JAMA Cardiol. 2020;5(7):811-8.

17. Shi $Q$, Zhang $X$, Jiang F, Zhang $X$, Hu N, Bimu C, et al. Clinical Characteristics and Risk Factors for Mortality of COVID-19 Patients with Diabetes in Wuhan, China: A Two-Center, Retrospective Study. Diabetes Care. 2020;43(7):1382-91.

18. Wang X, Fang J, Zhu Y, Chen L, Ding F, Zhou R, et al. Clinical characteristics of non-critically ill patients with novel coronavirus infection (COVID-19) in a Fangcang Hospital. Clin Microbiol Infect. 2020;26(8):1063-8.

19. Yan $Y$, Yang $Y$, Wang $F$, Ren $H$, Zhang S, Shi X, et al. Clinical characteristics and outcomes of patients with severe covid-19 with diabetes. BMJ Open Diabetes Res Care. 2020;8(1):e001343.

20. Yang JK, Feng Y, Yuan MY, Yuan SY, Fu HJ, Wu BY, et al. Plasma glucose levels and diabetes are independent predictors for mortality and morbidity in patients with SARS. Diabet Med. 2006;23(6):623-8.

21. Alqahtani FY, Aleanizy FS, Ali El Hadi Mohamed R, Alanazi MS, Mohamed N, Alrasheed MM, et al. Prevalence of comorbidities in cases of Middle East respiratory syndrome coronavirus: a retrospective study. Epidemiol Infect. 2018;147:1-5. 
22. Banik GR, Alqahtani AS, Booy R, Rashid H. Risk factors for severity and mortality in patients with MERS-CoV: Analysis of publicly available data from Saudi Arabia. Virol Sin. 2016;31(1):81-4.

23. Wang W, Chen H, Li Q, Qiu B, Wang J, Sun X, et al. Fasting plasma glucose is an independent predictor for severity of $\mathrm{H} 1 \mathrm{~N} 1$ pneumonia. BMC Infect Dis. 2011;11:104.

24. Qin C, Zhou L, Hu Z, Zhang S, Yang S, Tao Y, et al. Dysregulation of immune response in patients with Coronavirus 2019 (COVID-19) in Wuhan, China. Clin Infect Dis. 2020;71(15):762-8.
25. Yang JK, Lin SS, Ji XJ, Guo LM. Binding of SARS coronavirus to its receptor damages islets and causes acute diabetes. Acta Diabetol. 2010;47(3):193-9.

26. Zhang Y, Geng X, Tan Y, Li Q, Xu C, Xu J, et al. New understanding of the damage of SARS-CoV-2 infection outside the respiratory system. Biomed Pharmacother. 2020;127:110195. 